University of Wollongong

Research Online

Faculty of Social Sciences - Papers (Archive) Faculty of Arts, Social Sciences \& Humanities

$1-1-2018$

Support to enhance level of implementation in physical activity interventions: An observational study

Karel Strooband

University of Wollongong, kfbs149@uowmail.edu.au

Rebecca M. Stanley

University of Wollongong, rstanley@uow.edu.au

Anthony D. Okely

University of Wollongong, tokely@uow.edu.au

Rachel A. Jones

University of Wollongong, rachelj@uow.edu.au

Follow this and additional works at: https://ro.uow.edu.au/sspapers

Part of the Education Commons, and the Social and Behavioral Sciences Commons

Research Online is the open access institutional repository for the University of Wollongong. For further information contact the UOW Library: research-pubs@uow.edu.au 


\title{
Support to enhance level of implementation in physical activity interventions: An observational study
}

\author{
Abstract \\ 2018, Early Childhood Australia Inc. All Rights reserved. THIS ARTICLE AIMS TO DESCRIBE the level of \\ implementation (LOI) of the Jump Start study, and examine the relationship between LOI changes and the \\ support types provided to early childhood education and care (ECEC) centres. Direct observations were \\ conducted in 21 ECEC centres at six months and 12 months after the start of the intervention. Each \\ centre's LOI percentage was calculated and classified into high, medium or low implementers using \\ objective criteria. Support strategies were determined by LOI at first observation. Pearson correlations \\ between the support types and LOI were calculated using SPSS Statistics (version 23.0). In most cases \\ (86\%), ongoing support led to an increase in LOI, $17.5 \%$ on average. Phone calls were the only type of \\ support significantly related to the positive LOI change $(r=0.532, p=0.013)$. It was difficult to detect \\ other effective support types due to the combined and small variance in support types provided to each \\ centre.

\section{Disciplines} \\ Education | Social and Behavioral Sciences

\section{Publication Details} \\ Strooband, K., Stanley, R., Okely, A. \& Jones, R. (2018). Support to enhance level of implementation in \\ physical activity interventions: An observational study. Australasian Journal of Early Childhood, 43 (1), \\ 25-33.
}




\title{
Support to enhance level of implementation in physical activity interventions: An observational study
}

\author{
Karel FB Strooband, Rebecca M Stanley, Anthony D Okely and Rachel A Jones \\ University of Wollongong
}

\begin{abstract}
This article aims to describe the level of implementation (LOI) of the Jump Start study and examine the relationship between LOI changes and the support types provided to early childhood education and care (ECEC) centres. Direct observations were conducted in 21 ECEC centres at six months and 12 months after the start of the intervention. Each centre's LOI percentage was calculated and classified into high, medium or low implementers using objective criteria. Support strategies were determined by LOI at first observation. Pearson correlations between the support types and LOI were calculated using SPSS Statistics (version 23.0). In most cases (86\%), ongoing support led to an increase in LOI, $17.5 \%$ on average. Phone calls were the only type of support significantly related to the positive LOI change $(r=0.532, p=0.013)$. It was difficult to detect other effective support types due to the combined and small variance in support types provided to each centre.
\end{abstract}

\section{Introduction}

Physical activity (PA) interventions in early childhood education and care (ECEC) settings have shown mixed results, with most interventions reporting only moderate effects on PA outcomes (Alhassan, Sirard \& Robinson, 2007; Bellows, Davies, Anderson \& Kennedy, 2013; Cardon, Labarque, Smits \& De Bourdeaudhuij, 2009; Fitzgibbon et al., 2006; O'Dwyer et al., 2013; Van Cauwenberghe, De Bourdeaudhuij, Maes \& Cardon, 2012; Ward, Vaughn, McWilliams \& Hales, 2010). A number of factors may be contributing to the modest changes, including study duration, length of follow-up, intervention approach and level of intervention fidelity (Ling, Robbins \& Wen, 2016).

Intervention fidelity, which includes information about the content, coverage, frequency and duration of all intervention elements (Carroll et al., 2007), is important as it reflects the 
quality and integrity of the intervention (i.e. is the program delivered as planned?) (Steckler \& Linnan, 2002). Despite the importance of such data, implementation fidelity data is rarely reported (Alhassan \& Whitt-Glover, 2014; Bellows et al., 2013; Jones et al., 2011; Reilly et al., 2006; Trost, Fees \& Dzewaltowski, 2008). In the few studies that have reported intervention fidelity, the methods detailing the intervention fidelity are often inadequately described. For example, Alhassan and Whitt-Glover (2014) provided some information about their intervention fidelity but did not adequately describe their intervention fidelity processes. The study by Trost et al. (2008) is one of the few that clearly described how intervention fidelity data was assessed and what support was offered to educators. Intervention fidelity percentages were calculated using checklists, which were purposively developed for the study and completed by educators. In their study, a high level of implementation was recorded when their checklist was used. However, when these levels were compared with independent classroom observations, the level of implementation was not consistent (i.e. high implementation was recorded using the checklists but low implementation was recorded by the independent observer). To ensure that implementation was actually high, Trost et al. (2008) offered additional support to the educators during the implementation period. Adequate reporting of implementation data might help to identify strategies to increase intervention fidelity (Tate et al., 2016; Ward et al., 2010).

Methods to increase intervention fidelity have been varied, and few have been assessed. One possible method of ensuring high intervention fidelity might be through the provision of ongoing contextualised support for those delivering the intervention. Different types of support have been explored in PA interventions within ECEC settings, including professional development for educators (Jones et al., 2011; Pate et al., 2016; Pfeiffer et al., 2013; Reilly et al., 2006; Trost et al., 2008), booster sessions and individual feedback (Trost et al., 2008), site support visits and newsletters (Pfeiffer et al., 2013). However, such support has been intermittent or provided in a one-off session (Jones et al., 2011; Pate et al., 2016; Pfeiffer et al., 2013; Reilly et al., 2006; Trost et al., 2008). Ongoing support, where educators are in regular contact with researchers involved in the study, may be a more favourable method of increasing intervention fidelity and may, in turn, result in more meaningful and significant changes in outcomes. Given the dearth of studies that have investigated the influence of the 
type and level of support provided to centres on fidelity of implementation, the aims of this study were two-fold: (1) to describe the level of intervention implementation of the Jump Start study at two points throughout the intervention period; and (2) to examine the relationship between the types of support provided to centres and changes in intervention fidelity. It was hypothesised that the level of ongoing support would be directly associated with increasing implementation levels, and as such the fidelity of the intervention.

The theoretical framework for the Jump Start Intervention has been previously described (Stanley et al., 2016). In summary, the intervention is based on Bandura's Social Cognitive Theory (Bandura, 2004), in which four key learning processes (attention, retention, production and motivation) were followed and adopted within the Jump Start components to enhance behaviour change for educators, parents and young children (Bandura, 1986).

\section{Method}

\section{Design and participants}

Methods of the Jump Start study have been previously published (Stanley et al., 2016). Briefly, Jump Start is an 18-month randomised controlled trial implemented in 43 ECEC centres across New South Wales, Australia. Twenty-two ECEC centres were randomised to the intervention (Jump Start approach) and 21 ECEC settings were randomised to the comparison group (usual practice). Jump Start consists of five components: Jump In, Jump Out, Jump Up, Jump Through and Jump Home. Jump In is a 20-minute structured PA lesson, which focuses on developing gross motor skills. Jump Out focuses on additional practice time of the gross motor skill learnt in the Jump In lessons. The Jump Up activities aim to break up sitting time through high energy breaks involving music and dance elements. The Jump Through component incorporates routine learning experiences with PA, for example in children's group-time or transitions (i.e. time between structured activities such as between group time and meal time). The Jump Home component encourages parents to engage in the Jump Start activities in the home environment. The primary outcome was the time spent in total physical activity over a usual day during ECEC centre hours, measured by accelerometry (Actigraph GT3X+) and was assessed at baseline, six months and 18 months. Gross motor skill development, weight status, bone strength, self-regulation and parent and 
educator self-efficacy were secondary outcomes that were assessed at baseline and 18 months only (Stanley et al., 2016).

\section{Ethics, consent and funding}

The University of Wollongong Human Research Ethics Committee approved all study procedures in May 2014 (HE14/137). Latest approval was given in May 2016. Children's parents and educators were provided information packages and a recruitment video detailing the research and ethical requirements. Children's parents and educators had the opportunity to ask the research team any questions prior to providing written informed consent to participate in the study and to have their data published. This study was funded by a National Health and Medical Research Council (NHMRC) Project Grant (2014-2017; ID1062433). The funding body played no role in the study design; data collection, analysis, and interpretation; in the writing of the manuscript; or in the decision to submit the manuscript for publication.

\section{Process evaluation}

Process evaluation data was collected throughout the intervention period and included assessment of intervention fidelity at three time points (i.e. every six months). Intervention fidelity involved day-long observations and was assessed by trained personnel within the research group (i.e. research assistants) at six months, 12 months and 18 months using a study-specific direct observation tool. The observation tool involved recording start and finish times of prescribed Jump Start components, number of children (three- to five-yearolds) involved in each component, adherence to structured lesson plans, description of activities, use of equipment and resources, staff behaviours and additional comments (e.g. weather and environmental changes). For example, the Jump In questions reflected the activities from the lessons cards (e.g. Did the children explore catching different objects that are heavy or light [Yes/No]?), including exploration, guided discovery, reflection on learning, skill activities and additional questions (i.e. equipment use and staff behaviour). This study reports on data from the first and second observations (i.e. six and 12 months). Prior to the observations, research assistants attended a two-day intensive workshop, which focused on familiarisation of the direct observation tool, strategies for effective observations, child 
development information and practice conducting direct observations in two ECEC centres. The workshop was run by expert early childhood researchers and an interventionist with previous early childhood education and care experience as both a director and educator. Inter-observer reliability was assessed as part of the training (Hallgren, 2012). On the day of observation, data collectors positioned themselves in a non-conspicuous location within the ECEC environment. Observations were made throughout one whole day, depending on opening and closing times for each ECEC. Shortly after the observation day, research personnel deliberated on all components and external factors that could have been influencing the fidelity for each ECEC.

\section{Intervention fidelity}

Each centre's level of implementation (LOI) was calculated to access the intervention fidelity. The LOI was calculated using data from the Jump Start components that were implemented in the ECEC centre (i.e. Jump In, Jump Up, Jump Out and Jump Through). Each component was evenly weighted out of $25 \%$. A total of $25 \%$ was recorded if that component was implemented as intended. For example, Jump Up involved at least two energy breaks throughout the day. Zero per cent was given if no Jump Ups were observed, $12.5 \%$ was allocated if only one Jump Up was observed and $25 \%$ was allocated if two or more Jump Ups were observed. A similar scoring system was utilised for the Jump Out and Jump Through components. Jump In was more complex as the structured lessons comprised of four components: component one (Exploration) was scored out of $20 \%$, component two (Guided Discovery) was scored out of $40 \%$, component three (Reflect on Learning) was scored out of $10 \%$ and, finally, component four (Activities) was scored out of $30 \%$. This score out of $100 \%$ was standardised to a score out of $25 \%$. ECEC centres were classified into high, medium and low implementers after the first direct observation. For this classification, the $95 \%$ confidence interval was used to create a lower (41.6\%) and upper (68.0\%) boundary. Centres were reclassified, based on their LOI, following the second observation.

\section{Inter-observer reliability}

Inter-observer reliability was assessed using a two-way mixed absolute agreement, and calculation of an interclass correlation coefficient (ICC). Five inter-observer reliability tests 
were performed and resulted in single ICC agreements of $72.4 \%, 84.2 \%, 84.6 \%, 90.6 \%$ and 97.6\%. After achieving an agreement higher than $90.0 \%$, the four observers went into ECEC centres by themselves.

\section{Support}

Different types of support were provided during the period between the first and second direct observations by personnel of the research team. Each ECEC was allocated a support person from the research team, all of whom had extensive knowledge of the early childhood education sector and support protocol. These consisted of feedback of data from the first direct observation, support visits, phone calls, an additional face-to-face meeting, newsletters, an optional professional learning training day and goal setting. Details for each of these are described below. The quantity of support provided to the ECEC centres was determined by the LOI of the first direct observation (i.e. centres with low and medium classification were provided with more frequent support than those classed as high implementers). The ongoing support processes aimed to maximise ECEC director and educator engagement and offer opportunities to troubleshoot as necessary.

\section{Types of support}

1. Feedback of data from the first direct observation: LOI scores were presented to each centre at a face-to-face feedback session (usually a staff meeting) after the first direct observation. Results from first direct observation were compared with the optimal Jump Start approach and the average of all Jump Start centres.

2. Support visits: Support visits were initiated when centres specifically asked (19\%) for extra explanation of Jump Start components. Support visits consisted of direct feedback and modelling of the Jump Start components by the interventionist to enhance educator skills.

3. Phone calls: All centres participated in regular phone calls between the first and second process evaluation rounds. The intention was that centres in the medium and low implementer groups (based on the first round of process evaluation data) received monthly phone calls while centres classified as high implementers were contacted every third month. The primary objective of the phone call was to exchange contextual information between research personnel and the director and/or educators (e.g. reflecting on Jump 
Start progress, goals and setting new goals). Secondly, research personnel used phone calls to motivate directors and educators to maintain their interest in the program and their progress. For several centres, an additional face-to-face visit was provided with the same aim as a phone call.

4. Newsletters: A maximum of three newsletters were emailed and posted to the centres between the first and second process evaluations. The newsletters provided helpful learning examples of the Jump Start components.

5. Professional learning training day: Educators were given an opportunity to enrol in an intensive $(n=9)$ or refresher $(n=3)$ Jump Start training, eight months after the start of the intervention (free of charge). The intensive training involved a six- to eight-hour day that covered all Jump Start components in depth (e.g. philosophy, content, practice, integration and reflection on Jump Start components). This was targeted at educators who had not participated in the first intensive training before the intervention started. The refresher training was a two-hour, highly interactive session, which focused on reflection on current practices and enhancing performance of the Jump Start skills.

6. Goal setting: During the feedback session and the phone calls, educators were encouraged to set two or three SMART goals, which could be achieved prior to the next support opportunity. A total of 53 goals were set and ranked using the ranking system established by Hammersley et al. (Under Review). Briefly, all five SMART elements (Specific, Measurable, Attainable, Relevant and Timely) were coded ' 0 ' for non-applicable and ' 1 ' for applicable. Total scores were presented in a ranking scale from zero to five (Hammersley et al., Under Review).

\section{Statistical analyses}

Statistical analyses were completed using SPSS Statistics (version 23.0). Means and standard deviations were calculated with Excel and SPSS Statistics. T-tests were performed to create LOI boundaries, using the $95 \%$ confidence interval of the difference. Pearson correlations were conducted to examine the relationship between the LOI growth and the types of support. Statistical significance was set at $p \leq 0.05$. 


\section{Results}

\section{Centres}

One ECEC centre was excluded due to not wanting a direct observation being conducted in their centre at the second process evaluation. Therefore, data from 21 ECEC centres was used for analyses.

\section{Implementation scores}

Following the first direct observation, the average level of implementation was $54.8 \% \pm$ $29.0 \%$, with seven centres classified as high implementers (mean LOI $84.8 \% \pm 12.0 \%$ ), eight as medium implementers (mean LOI $55.6 \% \pm 7.0 \%$ ) and six as low implementers (mean LOI $18.4 \% \pm 15.9 \%)$, outlined in Figure 1. On average, less than half of the Jump In $(9.6 \%$ of $25 \%)$ and Jump Out (11.3\% of $25 \%$ ) components were being implemented as intended, and slightly more than half of the Jump Up and Jump Through components (16.1\% and $17.9 \%$ of $25 \%$, respectively) were being completed as intended at the first direct observation.

\section{$<$ Insert Figure 1 about here >}

Results from the second observations showed, on average, an increase in the level of implementation by $17.5 \%$. The number of components completed as intended for each individual element of the intervention increased between $2.9 \%$ and $6.6 \%$ : the number of Jump In components increased by $3.1 \%$ (from 9.6\% to $12.7 \%$ ); the number of completed Jump Out components increased by $6.6 \%$ (from $11.3 \%$ to $17.9 \%$ ); the number of Jump Up components increased by $4.7 \%$ (from $16.1 \%$ to $20.8 \%$ ); the number of Jump Through components increased by $2.9 \%$ (from $17.9 \%$ to $20.8 \%$ ). All implementation results for each ECEC centre and the four Jump Start components are presented in Table 1.

\section{$<$ Insert Table 1 here >}

Based on the grouping established following the first direct observation (using 95\% confidence intervals), four centres were reclassified from medium to high implementers (centres 8, 10, 11 and 14), five centres in the low group classification were reclassified as medium implementers (centres 16, 17, 18, 20 and 21) and one centre was reclassified from 
a low implementer to a high implementer (centre 19). Three centres had lower implementation scores following the second direct observation and two were reclassified: one from a high to a medium implementer (centre 3 ) and one from a medium to a low implementer (Centre 9).

\section{Support specifics}

The mean time between first and second direct observation was $172.5 \pm 35.8$ days. Support provided for each group (i.e. high, medium and low implementers) is presented in Table 2.

\section{$<$ Insert Table 2 here >}

Between the first and second direct observations: $100 \%$ of centres received feedback from their first direct observation outcomes, 19\% received a support visit, 53\% received support phone calls from researchers, $10 \%$ of centres participated in a face-to-face meeting, $38 \%$ of centres were involved in additional training, and $76 \%$ of centres participated in goal setting. There was a variation in quantity of newsletters due to the period between first and second direct observations; each centre received at least one with a maximum of three newsletters. The time between first direct observation and feedback ranged from 42 days to 181 days, with a mean of $116.8 \pm 46.1$ days. The time between feedback and the second direct observation varied between 19 and 92 days with an average of $55.3 \pm 27.5$ days. There was one outlier of 139 days (ECEC 18)-this centre was given feedback from their first observation before school holidays in 2015 , which was particularly early compared to the other centres. In total, 53 SMART goals were set with an average quality of $3.4 \pm 1.1$ (maximum score of five), and $57 \%$ reached the aim to set at least three goals, $19 \%$ of centres set one or two goals and $24 \%$ of centres set no goals.

\section{Correlations}

Table 3 shows the correlations between the change in LOI and support strategy. The number of phone calls was moderately related to the change in LOI $(r=0.53, p=0.013)$. No other significant correlations were found between support strategies and LOI changes. The 
implementation scores of the first direct observation were strongly associated to the changes in LOI $(r=-0.80, p<0.001)$.

\section{$<$ Insert Table 3 here >}

\section{Discussion}

This is one of the few studies that examined PA intervention fidelity of an ECEC setting in detail, and the relationship between intervention fidelity and ongoing support. Intervention fidelity increased over time as a result of ongoing support, with regular phone calls as the most important strategy of support.

Carroll at el. (2007) suggest that high intervention fidelity is key for the success of the intervention. In this study, six of the seven centres that were classified as high implementers after the first evaluation, retained their rating following the second observation. It is possible that in these centres a 'ceiling effect' occurred (Jones et al., 2014), whereby the support offered to these centres was intentionally not as substantial as that offered to other centres (Jones et al., 2015). In the centres where implementation levels remained high, Jump Start was embedded into the centre's routine. Furthermore, in these centres most educators were trained in the Jump Start approach, which meant that implementation did not rely on one or two educators but the load was shared between all educators.

Additionally, in these centres, the support from their directors was noticeably apparent and educators were given credit from their directors for their involvement in the Jump Start approach.

As perhaps expected, the centres that were rated low and medium following the first observation had greater room for improvement, and the support offered to these centres may have been perceived as more meaningful. This is supported by the strong correlation between implementation scores at the first observation and their changes of LOI $(r=-0.80$, $p<0.001)$. For these centres, a number of factors may have contributed to the intervention implementation increases. For example, a number of educators from these centres anecdotally reported changes in children's behaviour, confidence and self-regulation, which 
may have, in turn, encouraged further regular implementation of Jump Start. In some centres, receiving the feedback from the first observation was helpful in motivating them to increase their implementation level and perform better at the second observation. A number of centres mentioned that they were surprised at their implementation level at the first observation and wanted to make changes to ensure that their implementation level was much higher. Similar to the high performing centres, for some of these centres the director's support was also valuable in increasing implementation levels. In addition to the anecdotal evidence from educators, another important factor is likely to be the support received from the research team (i.e. provision of newsletters, phone calls, etc.) and the relationship built between educators and researchers (Jones, Gowers, Stanley \& Okely, 2017).

Phone calls were found to have positive effects on the LOI. No other studies have investigated the impact of phone calls on levels of implementation, however in a study with adults, regular phone calls were helpful in monitoring behaviour change (Hammersley, Cann, Parrish, Jones \& Holloway, 2015). Hammersley et al. (2015) showed that in a weightrelated telephone intervention, participants who received regular phone calls from the research team reported enhanced weight loss, positive changes in body mass index and increased time spent engaging in moderate- to vigorous-intensity physical activity. Similar to their phone calls, the phone calls in this study were contextualised for each centre. That is, questions pertaining to successes and barriers were asked specifically for each centre. This meant that each phone conversation, although it followed a general script, was different from the next and intentionally bespoke. Specifically, contextualising the support for each centre may have been a significant contributor to the changes observed in the implementation levels.

Other types of support, such as the support visits, may also have been important in changing intervention implementation. Although, in this study, support visits did not show a significant correlation with the LOI, they have been reported as beneficial in other studies. Howie et al. (2014) implemented a similar study (SHAPES) in preschool-aged children. Their study included support mechanisms such as educator training and workshops, newsletters and on-site visits. Their on-site visits had similar intentions to the support visits in this study 
but were more frequent than the current study (three per month in first year, two per month in the second year and up to seven in the third year). Although more frequent than those offered in the current study, the on-site visits were considerably shorter in duration than those in this study ( \pm 40 minutes vs $120-300$ minutes). Despite frequency and duration, their intent was similar to the current study and educators suggested that the site visits were important for increasing accountability, solving problems and recognising barriers (Howie, Brewer, Brown, Saunders \& Pate, 2016). The Jump Start intervention did not interview educators after the support visits, however, conversations between the researchers and educators suggest that the support visits were helpful in maintaining accountability in terms of intervention implementation.

Low implementers received more support visits $(n=3)$ compared with medium $(n=1)$ and high implementers $(n=0)$. However, this did not result in a significant change in LOI $(r=$ $0.376, p=0.093)$. For the centres that did receive a support visit, all improved their LOI at the second observation by an average of 33.2\% (range $21.4-61.0 \%)$. Support visits may have been beneficial to these centres, however, the small variation in the number of support visits provided may have prevented significant results from being reported. The effect of support visits on intervention implementation needs further investigation.

Of the 21 centres, three decreased their level of implementation from the first to the second observation. One (ECEC centre 2) only decreased by $3.4 \%$ (from $95.8 \%$ to $92.4 \%$ ) and was still classified as a high implementer at the second direct observation. The other two ECEC centres reduced their LOI by approximately 33\% (ECEC centre 3 [from 95.0\% to 62.1\%] and ECEC centre 9 [from 65.1\% to 32.8\%]). These centres dropped a level in implementation classification. Support data reveals that ECEC centre 3 received one newsletter and set three goals, but did not receive phone calls or visits, nor participated in training. Researchers tried to ring and visit this centre on a number of occasions, however educators were not available when times were set up. They were offered training, but chose not to participate in it. Conversations with the lead educator from this centre suggested that the decline was due to a 'bad day' on the second observation and re-organisation of the outdoor area, which minimised the outdoor play space. They implied that the second direct observation did not reflect a typical daily routine. For ECEC centre 9, one educator had participated in the 
refresher training and the centre received two newsletters. They did not receive phone calls or visits, nor participated in goal setting due to a relative high score at process evaluation one and the lack of time to participate in support. After the first process evaluation, only minimal support was deemed appropriate because their initial implementation scores were classified as high. Anecdotal evidence suggests that these centres may have relapsed to their previous habits at the second direct observation (e.g. providing a very controlled environment and only allowing one child at a time to participate in the activities). These habits were not the intention of the Jump Start intervention. Therefore, it is suggested that a low amount of support during this period in combination with external factors, such as a small outdoor area, low educator confidence (i.e. educator confidence in being able to implement Jump Start as intended) led to a declined intervention implementation. Additionally, in these centres, a number of staff changes were observed from the first observation to the second observation, which might have impacted the 'normal' day (e.g. children getting used to new educators and routines being disrupted), and ultimately a decline in intervention implementation. So, this indicates that both researchers and educators play an important role in the intervention fidelity, as also suggested by Steckler and Linnan (2002).

\section{Recommendations for further research}

To date, few physical activity interventions for preschool-aged children have adequately reported implementation fidelity. A variety of support strategies may be beneficial for implementation fidelity; however, further investigation of which types of support strategies are most effective is needed. Research around support strategies, frequency and types of support might reveal unique combinations that optimise intervention implementation. Understanding the best support strategy for PA interventions could enhance implementation and, in turn, improvements in the desired outcomes; however, increasing support strategies for PA interventions is costly and likely to be a lower priority for study budgets. Therefore, identifying the most efficient strategies will benefit study budgets and minimise unnecessary expenditures. 


\section{Limitations}

These findings need to be considered in light of the following limitations:

1. There are other factors that might influence implementation levels, for example, routines in centres and the motivation levels of educators. The Jump Start intervention commenced in July 2015, with the first direct observation being conducted in November-December 2015. The second direct observation was conducted in March-April 2016. Routines were firmly established in centres by the time the intervention was introduced and some educators were reluctant to change their routines. However, they were more willing to modify their routines to include the Jump Start components at the start of a new calendar year (i.e. at the start of 2016).

2. The direct observation was completed on one day. A non-typical day, poor weather or other external factors might incorrectly reflect what happens on a typical day at a centre.

Additionally, the Hawthorne effect may have played a part, where some centres tried to demonstrate more components due to the presence of the observer (Parsons, 1974). Multiple observation days or randomly assigned observation days are preferable. However, this was not possible for this study due to budgetary constraints.

3. Educators implementing the intervention have the most influence over the implementation fidelity. Some educators were not confident or showed resistance towards the philosophy of the intervention and thus did not prioritise implementing the different components of the intervention.

4. Due to the multiple components and complexity of the intervention, the observation tool used for the implementation fidelity was not standardised but specifically developed for this study. Thus, this tool can only be used for this study to reflect on the fidelity of the intervention.

\section{Conclusion}

The findings from this study suggest that specific ongoing support during midpoint intervention has a positive impact on the implementation fidelity, despite the possibility of multiple external factors potentially impacting changes in implementation fidelity. However, increased levels of implementation are only strongly related when the implementation of the intervention is low or medium at first measurement, possibly due to a 'ceiling effect'. The only support strategy that showed to be significantly related with the positive changes in level of implementation was phone calls. However, other strategies may be important as well. To date, there is very little evidence of implementation fidelity in the ECEC settings and 
more studies need to draw on existing evidence from other settings to explore what can be applied in these ECEC settings. Also, more research in support strategies is needed to understand if various support strategies are effective for increasing the intervention implementation and, in turn, enhance intervention outcomes.

\section{Acknowledgements}

The authors thank all preschool children, educators, directors and other staff members for participating in the study. Additionally, the authors acknowledge other members of the Early Start Research Institute research team involved in this study, Penny Cross and Dylan Cliff, especially Fay Gowers and Elisabeth-Ann Marshall for assisting in data collection.

\section{ORCID i/Ds}

Karel FB Strooband: 0000-0002-8719-0219

Rebecca M Stanley: 0000-0001-7007-3406

Anthony D Okely: 0000-0002-1626-8170

Rachel A Jones: 0000-0002-5384-1941

\section{References}

Alhassan, S., Sirard, J. R., \& Robinson, T. N. (2007). The effects of increasing outdoor play time on physical activity in Latino preschool children. International Journal of Pediatric Obesity, 2(3), 153-158.

http://dx.doi.org/10.1080/17477160701520108

Alhassan, S., \& Whitt-Glover, M. C. (2014). Intervention fidelity in a teacher-led program to promote physical activity in preschool-age children. Preventive Medicine, 69 (Supplement), S34-S36. https://doi.org/10.1016/j.ypmed.2014.07.024

Bandura, A. (1986). Social foundations of thought and actions. Englewood Cliffs, NJ: Prentice-Hall.

Bandura, A. (2004). Health promotion by social cognitive means. Health Education \& Behavior, 31(2), 143-164. https://doi.org/10.1177/1090198104263660

Bellows, L. L., Davies, P. L., Anderson, J., \& Kennedy, C. (2013). Effectiveness of a physical activity intervention for Head Start preschoolers: A randomized intervention study. The American Journal of Occupational Therapy, 67(1), 28-36. http://dx.doi.org/10.5014/ajot.2013.005777 
Cardon, G., Labarque, V., Smits, D., \& De Bourdeaudhuij, I. (2009). Promoting physical activity at the pre-school playground: The effects of providing markings and play equipment. Preventive Medicine, 48(4), 335-340. https://doi.org/10.1016/j.ypmed.2009.02.013

Carroll, C., Patterson, M., Wood, S., Booth, A., Rick, J., \& Balain, S. (2007). A conceptual framework for implementation fidelity. Implemention Science, 2(40). https://doi.org/10.1186/1748-5908-2-40

Fitzgibbon, M. L., Stolley, M. R., Schiffer, L., Van Horn, L., KauferChristoffel, K., \& Dyer, A. (2006). Hip-Hop to Health Jr. for Latino preschool children. Obesity, 14(9), 16161625.

Hallgren, K. A. (2012). Computing inter-rater reliability for observational data: An overview and tutorial. Tutorials in Quantitative Methods for Psychology, 8(1), 2334.

Hammersley, M. L., Cann, V. R., Parrish, A.-M., Jones, R. A., \& Holloway, D. J. (2015). Evaluation of the effects of a telephone-delivered health behaviour change program on weight and physical activity. Nutrition \& Dietetics, 72(4), 356-362. https://doi.org/10.1111/1747-0080.12213

Hammersley, M. L., Fisher, A., Jones, R. A., Morgan, P. J., Collins, C., \& Okely, A. D. (Under Review). Goal setting for weitght-related behavior change in children: an exploratory study. University of Wollongong.

Howie, E. K., Brewer, A., Brown, W. H., Pfeiffer, K. A., Saunders, R. P., \& Pate, R. R. (2014). The 3-year evolution of a preschool physical activity intervention through a collaborative partnership between research interventionists and preschool teachers. Health Education Research, 29(3), 491-502. https://doi.org/10.1093/her/cyu014

Howie, E. K., Brewer, A. E., Brown, W. H., Saunders, R. P., \& Pate, R. R. (2016). Systematic dissemination of a preschool physical activity intervention to the control preschools. Evaluation and Program Planning, 57, 1-7. https://doi.org/10.1016/j.evalprogplan.2016.03.006

Jones, J., Wolfenden, L., Wyse, R., Finch, M., Yoong, S. L., Dodds, P., . . Wiggers, J. (2014). A randomised controlled trial of an intervention to facilitate the implementation of healthy eating and physical activity policies and practices in childcare services. BMJ Open, 4(4), e005312. http://dx.doi.org/10.1136/bmjopen-2014-005312 
Jones, J., Wyse, R., Finch, M., Lecathelinais, C., Wiggers, J., Marshall, J., ... Wolfenden, L. (2015). Effectiveness of an intervention to facilitate the implementation of healthy eating and physical activity policies and practices in childcare services: a randomised controlled trial. Implementation Science, 10(147). https://doi.org/10.1186/s13012-015-0340-z

Jones, R. A., Riethmuller, A., Hesketh, K., Trezise, K., Batterham, M., \& Okely, A. D. (2011). Promoting fundamental movement skill development and physical activity in early childhood settings- a cluster randomized controlled trail. Pediatric exercise science, 23(4), 600-615.

Jones, R. A., Gowers, F., Stanley R. M., \& Okely, A. D. (2017). Enhancing the effectiveness of early childhood educators and researchers working together to achieve common aims. Australasian Jorunal of Early Childhood, 42(1), 81-84.

Ling, J., Robbins, L. B., \& Wen, F. (2016). Interventions to prevent and manage overweight or obesity in preschool children: A systematic review. International Journal of Nursing Studies, 53, 270-289. https://doi.org/10.1016/j.ijnurstu.2015.10.017

O'Dwyer, M. V., Fairclough, S. J., Ridgers, N. D., Knowles, Z. R., Foweather, L., \& Stratton, G. (2013). Effect of a school-based active play intervention on sedentary time and physical activity in preschool children. Health Education Research, 28(6), 931-942. https://doi.org/10.1093/her/cyt097

Parsons, H. M. (1974). What Happened at Hawthorne? Science, 183(4128), 922-932.

Pate, R. R., Brown, W. H., Pfeiffer, K. A., Howie, E. K., Saunders, R. P., Addy, C. L., \& Dowda, M. (2016). An intervention to increase physical activity in children: A randomized controlled trial with 4-year-olds in preschools. American Journal of Preventive Medicine, 51(1), 12-22. https://doi.org/10.1016/j.amepre.2015.12.003

Pfeiffer, K. A., Saunders, R. P., Brown, W. H., Dowda, M., Addy, C. L., \& Pate, R. R. (2013). Study of health and activity in preschool environments (SHAPES): Study protocol for a randomized trial evaluating a multi-component physical activity intervention in preschool children. BMC Public Health, 13(728).

Reilly, J. J., Kelly, L., Montgomery, C., Williamson, A., Fisher, A., McColl, J. H., . . Grant, S. (2006). Physical activity to prevent obesity in young children: Cluster 
randomised controlled trial. BMJ, 333(7577), 1041.

doi:10.1136/bmj.38979.623773.55

Stanley, R. M., Jones, R. A., Cliff, D. P., Trost, S. G., Berthelsen, D., Salmon, J., . . Okely, A. D. (2016). Increasing physical activity among young children from disadvantaged communities: study protocol of a group randomised controlled effectiveness trial. BMC Public Health, 16(1095). https://doi.org/10.1186/s12889-016-3743-0

Steckler, A., \& Linnan, L. (Eds.). (2002). Process evaluation for public health interventions and research. San Francisco, CA: Jossey-Bass.

Tate, D. F., Lytle, L. A., Sherwood, N. E., Haire-Joshu, D., Matheson, D., Moore, S. M., ... Michie, S. (2016). Deconstructing interventions: Approaches to studying behavior change techniques across obesity interventions. Translational Behavvioral Medicine, 6(2), 236-243. https://doi.org/10.1007/s13142-0150369-1

Trost, S. G., Fees, B., \& Dzewaltowski, D. (2008). Feasibility and efficacy of a 'move and learn' physical activity curriculum in preschool children. Journal of Physical Activity and Health, 5(1), 88-103.

Van Cauwenberghe, E., De Bourdeaudhuij, I., Maes, L., \& Cardon, G. (2012). Efficacy and feasibility of lowering playground density to promote physical activity and to discourage sedentary time during recess at preschool: A pilot study. Preventive Medicine, 55(4), 319-321. https://doi.org/10.1016/j.ypmed.2012.07.014

Ward, D. S., Vaughn, A., McWilliams, C., \& Hales, D. (2010). Interventions for increasing physical activity at child care. Medicine \& Science in Sports \& Exercise, 42(3), 526534. https://doi.org/10.1249/MSS.0b013e3181cea406 
Table 1. Implementation scores for each ECEC centre

\begin{tabular}{|c|c|c|c|c|c|c|c|c|c|c|c|c|}
\hline \multirow[t]{2}{*}{ ECEC } & \multicolumn{3}{|c|}{ Jump In (\%) } & \multicolumn{3}{|c|}{ Jump Up (\%) } & \multicolumn{3}{|c|}{ Jump Through (\%) } & \multicolumn{3}{|c|}{ Jump Out (\%) } \\
\hline & OBV. 1 & OBV. 2 & Growth & OBV. 1 & OBV. 2 & Growth & OBV. 1 & OBV. 2 & Growth & OBV. 1 & OBV. 2 & Growth \\
\hline 1 & 22.5 & 23.8 & 1.3 & 25.0 & 25.0 & 0.0 & 25.0 & 25.0 & 0.0 & 25.0 & 25.0 & 0.0 \\
\hline 2 & 20.8 & 17.4 & -3.4 & 25.0 & 25.0 & 0.0 & 25.0 & 25.0 & 0.0 & 25.0 & 25.0 & 0.0 \\
\hline 3 & 20.0 & 12.1 & -7.9 & 25.0 & 25.0 & 0.0 & 25.0 & 12.5 & -12.5 & 25.0 & 12.5 & -12.5 \\
\hline 4 & 9.9 & 12.6 & 2.7 & 25.0 & 25.0 & 0.0 & 25.0 & 25.0 & 0.0 & 25.0 & 25.0 & 0.0 \\
\hline 5 & 5.8 & 8.3 & 2.5 & 25.0 & 25.0 & 0.0 & 25.0 & 25.0 & 0.0 & 25.0 & 25.0 & 0.0 \\
\hline 6 & 20.1 & 24.3 & 4.2 & 12.5 & 25.0 & 12.5 & 12.5 & 12.5 & 0.0 & 25.0 & 12.5 & -12.5 \\
\hline 7 & 6.7 & 17.6 & 10.9 & 25.0 & 25.0 & 0.0 & 25.0 & 25.0 & 0.0 & 12.5 & 25.0 & 12.5 \\
\hline 8 & 3.6 & 25.0 & 21.4 & 12.5 & 25.0 & 12.5 & 25.0 & 12.5 & -12.5 & 25.0 & 25.0 & 0.0 \\
\hline 9 & 15.1 & 7.8 & -7.4 & 12.5 & 0.0 & -12.5 & 25.0 & 25.0 & 0.0 & 12.5 & 0.0 & -12.5 \\
\hline 10 & 0.0 & 2.9 & 2.9 & 25.0 & 25.0 & 0.0 & 25.0 & 25.0 & 0.0 & 12.5 & 25.0 & 12.5 \\
\hline 11 & 16.8 & 25.0 & 8.2 & 12.5 & 25.0 & 12.5 & 25.0 & 25.0 & 0.0 & 0.0 & 25.0 & 25.0 \\
\hline 12 & 12.5 & 17.5 & 5.0 & 12.5 & 25.0 & 12.5 & 12.5 & 0.0 & -12.5 & 12.5 & 12.5 & 12.5 \\
\hline 13 & 0.0 & 14.3 & 14.3 & 12.5 & 12.5 & 0.0 & 25.0 & 25.0 & 0.0 & 12.5 & 12.5 & 12.5 \\
\hline 14 & 0.0 & 1.9 & 1.9 & 25.0 & 25.0 & 0.0 & 25.0 & 25.0 & 0.0 & 0.0 & 25.0 & 25.0 \\
\hline 15 & 11.8 & 8.4 & -3.4 & 25.0 & 12.5 & -12.5 & 12.5 & 25.0 & 12.5 & 0.0 & 12.5 & 12.5 \\
\hline 16 & 11.3 & 1.0 & -10.3 & 25.0 & 25.0 & 0.0 & 0.0 & 12.5 & 12.5 & 0.0 & 25.0 & 25.0 \\
\hline 17 & 7.9 & 6.3 & -1.6 & 0.0 & 25.0 & 25.0 & 25.0 & 25.0 & 0.0 & 0.0 & 0.0 & 0.0 \\
\hline 18 & 0.0 & 3.8 & 3.8 & 12.5 & 12.5 & 0.0 & 12.5 & 25.0 & 12.5 & 0.0 & 25.0 & 25.0 \\
\hline 19 & 15.9 & 13.8 & -2.1 & 0.0 & 25.0 & 25.0 & 0.0 & 25.0 & 25.0 & 0.0 & 12.5 & 12.5 \\
\hline 20 & 0.0 & 12.8 & 12.8 & 0.0 & 0.0 & 0.0 & 0.0 & 25.0 & 25.0 & 0.0 & 12.5 & 12.5 \\
\hline 21 & 0.0 & 11.0 & 11.0 & 0.0 & 25.0 & 25.0 & 0.0 & 12.5 & 12.5 & 0.0 & 12.5 & 12.5 \\
\hline Mean & 9.6 & 12.7 & 3.1 & 16.1 & 20.8 & 4.7 & 17.9 & 20.8 & 2.9 & 11.3 & 17.9 & 6.6 \\
\hline
\end{tabular}

OBV: Observation 
Table 2. Support provided for high, medium and low level of implementation centres between the first and second direct observation

\begin{tabular}{lllll}
\hline Support strategies & High & Medium & Low & All \\
& $n=7(\%)$ & $n=8(\%)$ & $n=6(\%)$ & $n=21(\%)$ \\
\hline Feedback session & $7(100)$ & $8(100)$ & $6(100)$ & $21(100)$ \\
\hline Support visits & $0(0)$ & $1(13)$ & $3(50)$ & $4(19)$ \\
\hline Number of phone call & & & & \\
One phone call & $1(14)$ & $3(38)$ & $5(83)$ & $9(43)$ \\
Two phone calls & $0(0)$ & $1(13)$ & $1(17)$ & $2(10)$ \\
\hline Additional F2F* visit & $1(14)$ & $1(13)$ & $0(0)$ & $2(10)$ \\
\hline Number of newsletter & & & \\
One newsletter & $2(29)$ & $4(50)$ & $1(17)$ & $7(33)$ \\
Two newsletters & $3(42)$ & $2(25)$ & $4(67)$ & $9(43)$ \\
Three newsletters & $2(29)$ & $2(25)$ & $1(17)$ & $5(24)$ \\
\hline Number of educators followed training & & \\
One educator & $1(14)$ & $2(25)$ & $2(33)$ & $5(24)$ \\
Two educators & $1(14)$ & $1(13)$ & $0(0)$ & $2(10)$ \\
Three educators & $0(0)$ & $0(0)$ & $0(0)$ & $0(0)$ \\
Four educators & $0(0)$ & $1(13)$ & $0(0)$ & $1(5)$ \\
\hline Number of goals set & & & & \\
No goals & $0(0)$ & $3(38)$ & $2(33)$ & $5(24)$ \\
One or two goals & $2(29)$ & $1(13)$ & $1(17)$ & $4(19)$ \\
Three or more & $5(71)$ & $4(50)$ & $3(50)$ & $12(57)$ \\
goals & & & & \\
\hline
\end{tabular}

*F2F = Face-to-face

Table 3. Correlations between changes of implementation levels and support strategies $(n=21)$

Change Level of Implementation (LOI)

Pearson Correlation

Feedback/ F2F*

Support visits

Phone call

Newsletter

Training

Goals
$-0.117$

$-0.376$

0.532

$-0.034$

0.072

0.103
Significance

(2-tailed)

0.614

0.093

0.013

0.885

0.755

0.658

${ }^{*} \mathrm{~F} 2 \mathrm{~F}=$ Face-to-face 
Figure 1. Comparison of level of Implementation (LOI) at direct observation one and two

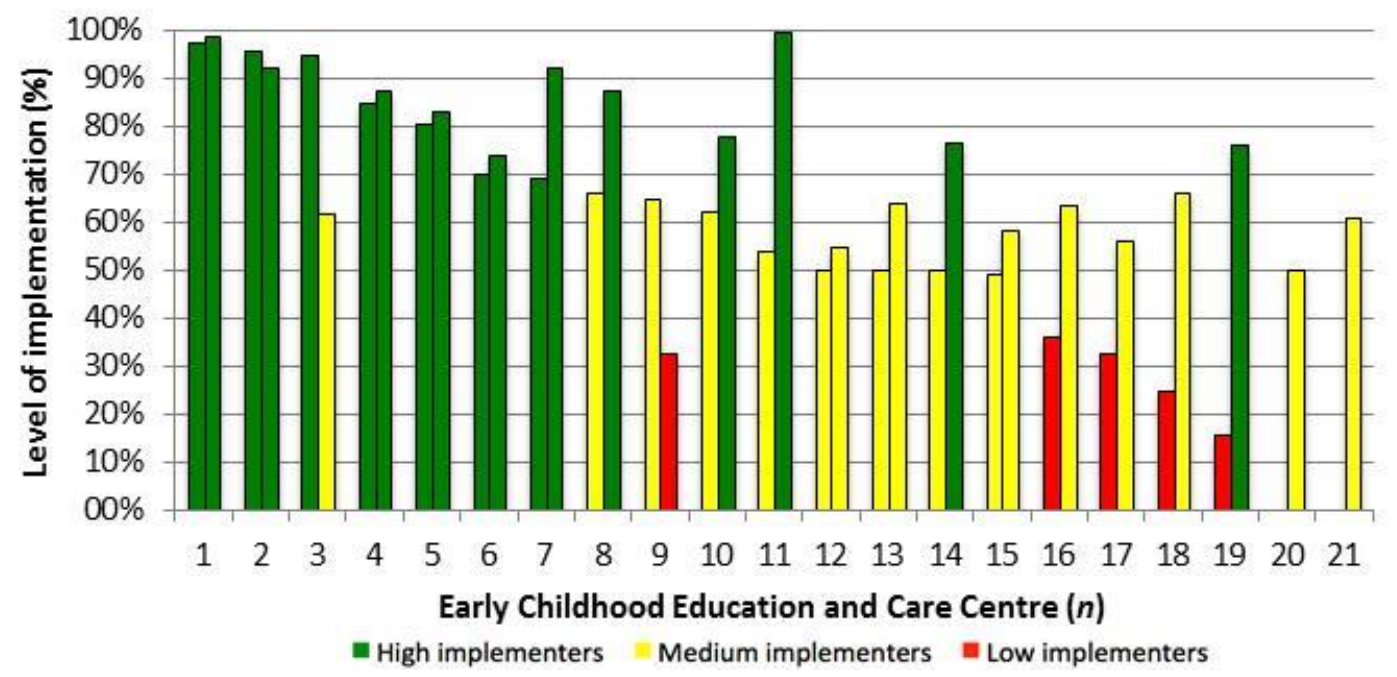

Note: First bar presents the implementation score of the first direct observation, second bar presents the implementation score of the second direct observation. 